\title{
Violence Syndicale Estudiantine Dans Les Universités Publiques De Côte d'Ivoire : Perceptions Et Enjeux
}

\author{
Diarassouba Ibrahima
}

Docteur en Sociologie, option politique.

Doctorat obtenu à l’Université Alassane Ouattara de Bouaké (Côte d'Ivoire).

doi: 10.19044/esj.2017.v13n7p379 URL:http://dx.doi.org/10.19044/esj.2017.v13n7p379

\begin{abstract}
During the decades 1990-2010 that followed the introduction of the multiparty system in 1990, the educational environment in particular the academic institutions in Côte d'Ivoire were greatly affected by the recurrence of student union violence. With the "new departure" which marks a new dynamics of the universities by a break with the practices which have begun their normal functioning based on the effect of giving them their luster from before, we have noted the survival of this violence. The aim of this study is to discover, on the one hand, the perception that student unionists have about violence and, on the other hand, to analyze the stakes involved in the systematic use of violence by these unionized students during the two decades of 1990- 2010 to understand the survival of this phenomenon in the context of "new departure". To achieve this, we have chosen the six (06) unions that have marked the history of student violence the more during the period 1990-2010. With the aid of semi-directive interviews, we have collected the data which reveal the perception that violence for the trade unions is a legitimate and legal act according to their own statute and internal regulations. It shows that there is a congenital link between trade unionism and politics. Therefore, trade unionism cannot be exercised without politics. Under the stakes, it appears that violence is an indicator of trade union dynamism for political positioning; that it confers power by the domination of public spaces such as universities.
\end{abstract}

Keywords: Syndicate, Violence, Political Positioning, Institutionalization, Resilience

\section{Résumé}

Durant les deux décennies (1990-2010) qui ont suivi l'instauration du multipartisme en 1990, le milieu éducatif notamment les établissements universitaires en Côte d'Ivoire ont fortement été perturbés par larécurrence 
des violences syndicales estudiantines. Avec le «départ nouveau » qui marque une nouvelle dynamique des universités par une rupture avec les pratiques qui ont entamé leur fonctionnement normal à l'effet de leur donner leur lustre d'antant, nous constatons la survivance de cette violence.La présente étude a pour objectif de découvrir d’une part, la perception que les étudiants syndicalistes ont de la violence, d'autre part, d'analyser les enjeux du recours systématique à la violence par ces étudiants syndicalistes durant les deux décennies 1990-2010 en vue de comprendre la survivance de ce phénomène dans le contexte de «départ nouveau ». Pour y parvenir, nous avons choisi les six (06) syndicats qui ont plus marqué l'histoire de violence estudiantine durant la période 1990-2010. A l'aide d'entretien semi-directif nous avons recueillie les données qui laissent apparaitre au titre de la perception, que la violence est pour les syndicats un acte légitime et légal conformément à leur propre statut et règlement intérieur; qu’il existe un lien congénital entre le syndicalisme et la politique, par conséquent, le syndicalisme ne peut s'exercer sans faire de la politique. Au titre des enjeux, il apparait que la violence est un indicateur du dynamisme syndical pour un positionnement politique ; qu’elle confère du pouvoir par la domination des espaces publics tels que les universités.

Mots clés: Syndicat, violence, positionnement politique, institutionnalisation, résilience.

\section{Introduction}

L'histoire de la mutation du syndicalisme estudiantin en violence extrême ne peut se conter sans la mettre en lien avec l'évolution sociopolitique de la Côte d'Ivoire, notamment son processus de démocratisation amorcé en 1990 avec l'instauration du multipartisme. Cette mutation politique qui marque un virage important de l'évolution politique de Côte d'Ivoire, est aussi une étape importante de l'histoire du syndicalisme estudiantin dans les universités publiques de la Côte d'Ivoire. Pour certains auteurs tels que Hyden, si l'année 1989 marquée par la chute du mur de Berlin doit être considérée comme l'année de l'Europe, l'année 1990 est celle de l’Afrique (Hyden, 1992). Ce processus démocratique amorcé en 1990 fut émaillé de vastes mouvements de contestation (N’goran \& Silué, 2102) qui n’ont pas épargnés le milieu éducatif notamment les universités publiques avec l'instauration d'un renouveau syndical par la fesci à partir de 1990. Ce renouveau syndical s’est manifesté dès le départ par le caractère contestataire de la lutte syndicale; la remise en cause permamente de l'autorité académique et administrative (N'goran, 2011; Vanga- Kouakou \& Sika, 2006) à travers les actes d'incivilité et l'atteinte à l'intégrité physique du personnel enseignant et administratif. Il s'est par la suite 
radicalisé avec d'une part, l'accoutumance des militants à l'usage des armes blanches tels que la machette, les faucilles, les couteaux etc désormais intégrés dans le mode opératoire des syndicats. D’autre part, cette radicalisation a favorisé la domination du champ universitaire et sa déstructuration en un lieu de rente du désordre par l'occupation anarchique des espaces pour l'exercice des activités génératrices de revenu ; l'instauration et le maintien d'un climat d'insécurité sur les campus et résidences unuversitaires. Ainsi, sous l'influence des mutations politiques, ce renouveau syndical a transformé les universités publiques en des lieux de déni de la démocratie syndicale ${ }^{59}$ (M’bra, 2010), des lieux d'exercice sans cesse continu de la violence estudiantine intra syndicale et intersyndicale (Goin Bi, 2010). Certains faits sociopolitiques qui ont accompagné la mutation politique en Côte d'Ivoire, ont influencés l'exercice du syndicalisme estudiantin. La création du front républicain en 1995 qui a favorisé une cohésion au sein de la fesci qui n’a cessé d'activer une logique oppositionnelle contre le parti démocratique de Côte d'Ivoire (PDCI) (Konaté, 2003). Sa dissolution en 1999 pour des désaccords politiques a accentué la division entre les militants de la fesci. Pour rappel, la crise des machettes survenue en 1998 à la fin du mandat de Soro Guillaume actuellement président de l'assemblée nationale, a opposé deux camps repartis entre le front populaire ivoirien (FPI) et le rassemblement des républicains (RDR). Chaque camp reprochait à l'autre de vouloir contrôler le syndicat au profit de son parti. Avec la rébellion de 2002 qui se présente comme la matérialisation politique de la crise des machettes, la violence se radicalise davantage. Cette micro-violence définie comme des expressions et actes de violence socialement et/ou géographiquement circonscrits (Mel, 2009) prend ainsi la dimension d'une macro-violence du fait de sa déportation hors du champ universitaire en allant des campus à la rue et aux espaces publics. Human Right Watch fait également ressortir dans son rapport annuel de 2008 la dimension macro de cette violence en montrant l'implication des écoles et des universités dans la crise militaro-politique qu'a vécue la Côte d'Ivoire, à travers la participation active de la fesci dans les violences politiques (HRW, 2008).

Avec le «départ nouveau » des universités amorcé à la rentrée universitaire du 03 septembre 2012, l’Etat dans sa volonté de redonner aux universités publiques leur lustre d'antant, a initié certaines reformes qui visent d'une part, à améliorer les conditions de travail et d'étude au sein des universités. D’autre part, à rompre avec les violences exercées par les syndicats d'étudiants qui ont considérablement entamées le bon

\footnotetext{
${ }^{59}$ Le refus de la liberté syndicale par la fesci qui s'opposait à l'emergence d'autres syndicats dans le but de maintenir son leadership
} 
fonctionnement des universités et détériorer la qualité de l'enseignement. Nonobstant ces reformes, nous constatons que la rupture recherchée par le « départ nouveau » n’a pu être effective avec la résurgence gravicime de la violence syndicale estudiantine, posant ainsi avec acquité la problématique du recours systématique et recurrent des étudiants syndicalistes à la violence. Il est important de rappeler à toute fin utile que le "départ nouveau » survient à la suite de deux décennies tumultueuses des universités publiques en Côte d'Ivoire. La recherche d'une nouvelle dynamique des unuiversités n’a pu être effective avec la résurgence de la violence d'une gravité extrême comme la continuté de la pratique des deux décennies précédentes. Comment comprendre alors la survivance de cette violence ? Cette interrogation nous amène à interroger la perception et les enjeux du recours systématique à la violence. La présente étude a donc pour objectif de découvrir d’une part, la perception que les étudiants syndicalistes ont de la violence, d'autre part, d'analyser les enjeux du recours systématique à la violence par les syndicats d'étudiants durant les deux décennies précédentes (1990-2010) en vue de comprendre la survivance de ce phénomène dans le contexte de "départ nouveau ». Ce qui justifie la circonscription de notre étude dans cette période. Pour répondre à cette interrogation, notre réflexion s'appuie sur l'approche phénoménologique qui nous permet de partir des discours des auteurs clés de la violence et tenir compte de la perception qu'ils ont de leurs actions. Ensuite, à partir de l'analyse de contenu de ces discours, nous montrons que la violence est un indicateur du dynamisme syndical pour un positionnement politique ; qu'elle confère du pouvoir par la domination des espaces publics tels que les universités.

Pour mieux rendre compte, l'étude s'articule autour:

$>$ De la présentation du champ syndical estudiantin et le fonctionnement des syndicats.

$>$ De la violence syndicale estudiantine dans l'arène politique.

$>$ De la perception de la violence par les syndicats d'étudiants.

$>$ Des enjeux de la violence syndicale.

\section{I- Présentation du champ syndical estudiantin et le fonctionnement des syndicats}

1- Présentation du champ syndical estudiantin

Durant les décennies qui ont suivi le multipartisme en 1990, on enrégistrait plus d'une dizaine de syndicats d'élèves et étudiants qui, en dépit de leurs différences idéologiques ${ }^{60}$, avaient tous la même mission qui est la lutte pour le bien-être des élèves et étudiants. Parmi ces syndicats, nous dénombrons six (06) qui ont marqué l’histoire de la violence de 1990 à 2010,

${ }^{60}$ Certains sont de la gauche syndicale et d'autres se reclament de la droite syndicale. 
pour avoir été auteurs pour certains, etd’autres pour avoir été victimes. Par ce constat, nous avons cherché à comprendre ce qui oppose ces syndicats. A la suite des données recueillies auprès des enquêtés, les motifs d’opposition de ces syndicats que nous avons retenus comme critères de choix des syndicats sont présentés dans le tableau ci-dessous ${ }^{61}$.

Tableau 1. Critères de choix des syndicats

\begin{tabular}{|c|c|c|c|}
\hline & \multicolumn{3}{|c|}{ CRITERES de choix des syndicats } \\
\hline Syndicats & $\begin{array}{c}\text { auteurs de } \\
\text { violence }\end{array}$ & Syndicats opposés & $\begin{array}{c}\text { Syndicats supposés } \\
\text { proches des partis } \\
\text { politiques }\end{array}$ \\
\hline AGEECI & $\times$ & Opposé à la FESCI & $\begin{array}{c}\text { proche du RDR et des ex } \\
\text { FAFN }\end{array}$ \\
\hline CERAC & & Opposée à la FESCI & proche du PDCI \\
\hline FESCI & $\times$ & UNESCI, CERAC, & proche du FPI \\
& & $\begin{array}{c}\text { UEGECI, SYEECI, CEECI, } \\
\text { AGEECI }\end{array}$ & proche du RDR \\
\hline SYEECI & $\times$ & FESCI & proche du PDCI \\
\hline UEGECI & & FESCI & proche du PDCI \\
\hline UNESCI & $\times$ & Opposée à la FESCI &
\end{tabular}

Source: Diarassouba Ibrahima-Données d'enquête de la thèse, 2016

L'opposition ici se pose d'abord en termes d'approche et d'idéologie syndicale avant de se matérialiser en acte de violence. Tous les syndicats opposés à la fesci sont nés après elle, et ils ont voulu se démarquer d'elle en adoptant comme principale approche la non violence et le dialogue qui semble être en phase avec l'idéologie du PDCI. Le choix de ce slogan n'est pas fortuit face à la mauvaise presse de la fesci dans la société et auprès du régime (PDCI) au pouvoir. Par ce slogan aussi bien expressif qu'attrayant qu'il soit ces syndicats voulaient s'inscrire aux antipodes des actions de la fesci à l'effet de bénéficier d'une adhésion massive des élèves et étudiants à leur cause, mais aussi de bénéficier des faveurs des partis politiques. On se rend compte de l'implication d'autres acteurs extérieursnotamment la politique dans le fonctionnement des syndicats, soit à partir de connexionsentre ces acteurs et les syndicats d'étudiants, soit par des actions précises, comme cela apparait dans les propos de F.Y et M.A.

"Cette violence entre nous là a été suscitée, sinon alimentée par les hommes politiques. Parce que pour un syndicat, tu vas avoir l'argent où pour l'installation de tes sections si ce n'est pas un financement extérieur. Or nous savons tous qu'ici là les plus riches sont les hommes politiques ». (Extrait de l'entretien avec F.Y, en 2015)

${ }^{61}$ Ces syndicats seraient proches des partis politiques sur la base des informations recueillies auprès des enquêtés. 
"Comme je le disais tout à l'heure, la violence en interne comme en externe, ya toujours la politique dedans. Voilà pourquoi j'ai dit diviser pour mieu règner. Les politiciens cherchent toujours à nous diviser pour mieux nous contrôler. C'est pour cela à un certain moment la fesci était face à d'autres associations telles que l'ageeci, le syeeci tout ça, justement c'est ça. Ils ont été créées pour nous combattre. » (Extrait de l'entretien avec M.A en 2015)

Dans son étude réalisée en 2013, Search For Common Ground met en exergue l'implication très siginificative d'autres acteurs extérieurs dans l'exercice de la violence dans le milieu éducatif en Côte d’Ivoire.La figure ci-dessous montre bien les acteurs impliqués dans la violence en milieu scolaire et universitaire.

Figure 1. Facteurs d'exacerbation de la violence syndicale dans le milieu scolaire et universitaire

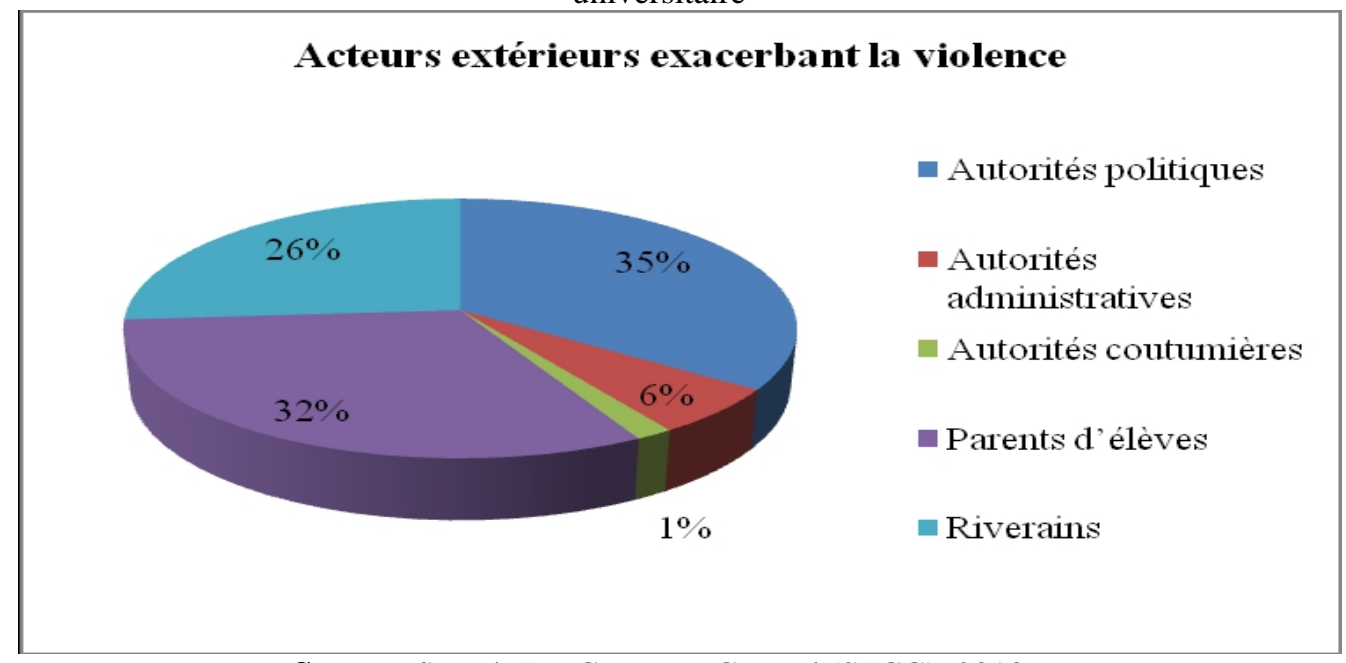

Source: Search For Common Ground (SFCG), 2013

Cette figure montre que la violence n’est pas un phénomène isolé. Son exacerbation est la conséquence de l'implication de plusieurs facteurs extérieurs avec une dominance du facteur politique.

\section{2- Fonctionnement des syndicats}

Au sein du bureau exécutif national, les décisions se prennent le plus souvent à l'unanimité au cours d'une réunion. Toutefois, le respect de cette procédure est conditionné par l'urgence et du niveau d'importance de la décision pour le syndicat. En effet, pour des situations d'urgence, le secrétariat général national prend la décision et la rend exécutoire par le secrétaire national à l'organisation. Toutes les décisions se prennent au cas par cas selon le niveau d'appréciation du secrétaire général. Les décisions une fois prises, les secrétariats à l'organisation se chargent de développer la 
stratégie de sa mise en œuvre dans le but de l'atteinte des résultats attendus. A la suite de nos entretiens avec nos enquêtés, il est à retenir que la procédure de prise de décisions au sein des syndicats est quasi identique.

"Les décisions chez nous se prennent de façon collégiale et exécutoire. Quand je dis collégiale ça veut dire que nous faisons comme le BEN, le SG pour prendre une décision il fait appel soit aux membres du bureau national pour statuer sur la situation, soit il organise une assemblée générale afin d'appliquer la décision de la majorité. Mais comprenez aussi qu'au-delà de tout, en tant que chef, tu es un élu donc tu jouis d'un pouvoir discrétionnaire qui te permet de décider aussi toi-même de façon exécutoire». (Extrait de l'entretien réalisé en 2015 avec B.L.R)

\section{II- La violence syndicale estudiantine dans l'arène sociale et politique}

1- La permanence du mouvement syndical estudiantin au cour des mouvements sociopolitiques en Côte d'Ivoire et la volonté du maintien d'un monolithisme syndical

A partir d'un rappel historique, M'bra nous apprend que le syndicalisme estudiantin s'est toujours inscrit au cœur des mouvements sociopolitiques en Côte d’Ivoire depuis la période coloniale. En effet, A la faveur du nouveau contexte politique instauré en Côte d'Ivoire le 7 août 1960 date de son accession à l'indépendance avec le PDCI au pouvoir, un second mouvement estudiantin voit le jour dénommé Union Nationale des Elèves et Etudiants de Côte d'Ivoire (UNECI). "L'objectif principal qui a sous-tendu la création de cette "Union" était de réhabiliter l'image de la Côte d'Ivoire quelque peu ternie par la FEANF ${ }^{62}$. Comme il fallait s'y attendre, les membres de l'UGECI ${ }^{63}$ ont traité ceux de l'UNECI de "corrompus" et d' "achetés". La discordance s'est donc instaurée entre les deux mouvements estudiantins. Pour le parti unique qui prône la pensée unique, deux mouvements estudiantins pour un jeune pays qui vient d'accéder à la magistrature suprême était à la fois trop et dangereux. Cette situation, en effet, pourrait fragiliser les efforts de construction nationale » (M’bra, op.cit : 5).

\footnotetext{
${ }^{62}$ Fédération des Etudiants d'Afrique Noire en France, proche du parti communiste français

${ }^{63}$ En 1948, fut créée l’Association Générale des Elèves et Etudiants de Côte d’Ivoire (AGEECI), avec une section en France. En vue d'être plus efficaces dans sa mission de défense des intérêts matériels et moraux des élèves et étudiants ivoiriens, mais surtout dans l'optique de ne pas se présenter aux hommes politiques de manière dispersée, les sections ivoirienne et française firent fusion. De cette fusion, naquit l'Union Générale des Elèves et Etudiants de Côte d'Ivoire (UGECI). Cette "Union" était affiliée à la FEANF.
} 
L’UNECI qui se limitait au milieu universitaire, va s'étendre au milieu scolaire en 1964 et devient UNEECI. Cette structure efficace de contrôle qui avait pour objectif de s'opposer aux mouvements de contestation menés par les étudiants ivoiriens basés en France dès les premières années de l'indépendance, se fait de plus en plus critique et engage un processus d'autonomisation vis-à-vis du PDCI à partir de 1967. Elle est donc dissoute en 1968 par le Bureau politique du PDCI qui l'accuse de faire office de parti d'opposition sous couvert du syndicalisme (Proteau, 1998). En 1969, le MEECI est créé par le PDCI en remplacement de l’UNEECI. Le MEECI qui devient une sous section du parti, servait de cadre de formation des futures élites politiques. Le maintien d'un monolithisme syndical pour exercer un contrôle idéologique sur la jeunesse estudiantine, crée une situation de frustration chez les étudiants contraints au mutisme. La violence syndicale estudiantine est donc un phénomène qui n’est pas né ex-nihilo. C'est un construit social qui trouve ses origines dans l'inexistence d'un cadre formel de libre expression des étudiants opposés au mode de gestion du PDCI. Cette situation du mutisme sous contrainte a eu pour conséquence l'instauration d'une tension sous couveuse qui demeura durant les trois décennies du système du parti unique. Pour ces étudiants, l'avènement du multipartisme était donc salutaire parce qu'il leurs permettrait de se défaire d'une part du joug du mutisme auquel ils étaient contraints, d'autre part de la tutelle politique en vue de leur autonomisation pour mieux revendiquer.

\section{2- L'instauration du multipartisme et la rupture du consensus politique sur fond d'aggravation de la crise financière de 1990 à 1993}

Les deux décennies qui après l'indépendance sont marquées par une stabilité sociopolitique matérialisée par un consensus politique. Ce consensus politique est la conséquence de la stabilité économique. Comme le fait remarquer Akindes (2000: 127), "sur le plan macro-économique, la dynamique politique de la côte d'Ivoire s'est déployée sur fond de dix huit années (1960-1978)». L'avènement du multipartisme en 1990, expression d'un renouveau démocratique s'est fait dans un contexte sociopolitique précaire résultant de la morositéde la situation économique.La réalisation effective de ce processus du renouveau démocratique a permis à la Côte d'Ivoire d'organiser sa première élection présidentielle ouverte et concurrentielle en octobre 1990 sanctionnée par la victoire d'Houphouët Boigny contre Laurent Gbagbo, candidat du FPI et figure charismatique de l'opposition politique. En dépit de la participation de l'opposition à cette élection, marquant l'effectivité du caractère concurrentiel et ouvert du multipartisme, ce nouvel ordre politique n'a toutefois pu garantir la stabilité politique en Côte d'Ivoire. Réputée pour sa stabilité politique au point 
qu'elle fut appelée « havre de paix », la Côte d'Ivoire a paradoxalement basculé dans l'instabilité sociopolitique. Le multipartisme perçu comme salvateur pour une stabilité sociopolitique en garantissant un processus démocratique ouvert, transparent, basé sur l'acceptation de la contradiction politique, n’a malheureusement pas permis d'y parvenir. Ce qui présageait déjà d'un processus démocratique manqué. Avec la persistance de la crise économique qui n’a pu être jugulée jusqu'en 1990, la rupture du consensus politique devient effective. Cette rupture commence au sein du PDCI en 1993 avec la guerre de succession après le décès du Félix Houphouët Boigny le 07 décembre 1993 entre Alassane Dramane Ouattara premier ministre d’alors et Henry Konan Bédié qui était président de l’assemblée nationale.

L'action politique, action par excellence historique est une expression de la liberté humaine dans l'histoire au sens où liberté est la condition de toute action, de toute politique (Draus, 1984). La manifestation de la liberté dans le nouvel ordre politique instauré par le multipartisme semble respecter cette logique. Du point de vue politique, la liberté d'expression, d'opinion et d'action induite par le multipartisme était de plus en plus effective avec la les partis politiques d'opposition qui étaient plus visibles. Toutefois, la manifestation de cette liberté a favorisé l'exacerbation des remous sociopolitiques suscités par une opposition politique bien structurée et de plus en plus critique de la gestion du pouvoir par Houphouët Boigny. Le nouvel ordre politique qualifié de renouveau démocratique instauré par le multipartisme ne s’est pas manifesté seulement dans le champ politique. Il s’est manifesté dans tous les secteurs. Dans le milieu syndical estudiantin, on assiste à la création de plusieurs syndicats d'étudiants sur le campus universitaires. Certains opposés au PDCI, d'autres qui lui servent de soutien. La fesci soutenue par le SYNARES se veut plus critique du pouvoir PDCI. Avec les changements constitutionnels induits par l'instauration du multipartisme, le poste de premier ministre est créé et Félix Houphouët Boigny fait appel à Alassane Dramane Ouattara, gouverneur de la Banque Centrale des Etats de l'Afrique de l'Ouest (BCEAO). L'occupation du poste de premier ministre par Alassane Dramane Ouattara a facilité le dialogue entre l'Etat de Côte d'Ivoire et les institutions de Bretton-Woods (Koné, 2011). Il dirige le pays jusqu’au décès du Président Houphouët Boigny en décembre 1993 dans un environnement marqué par le pluralisme politique et une grave crise économique qui n’a jusque là pu être jugulée. Cette crise est alimentée par les remous sociaux nés des effets impopulaires de la mise en œuvre des politiques d'ajustement structurel initiées par les institutions de Bretton-Woods. C'est le point de départ d'une longue période de tension sociale. Ce technocrate économiste propulsé au devant de la scène politique par cette nomination à la tête du gouvernement ivoirien, avec pour mission de mener des reformes en vue d'équilibrer les finances publiques et juguler 
la crise économique persistante devient par la suite un opposant politique dérangeant pour le PDCI. La guerre de succession pour la gestion de la transition après le décès d’Houphouët Boigny commence donc et crée une division au sein de la famille PDCI. Cette division a abouti à la désolidarisation de certains membres de la famille " pédécéiste » avec à leur tête Djéni Kobénan et Alassane Dramane Ouattara pour créer le Rassemblement Des Républicains (RDR) en 1994.

\section{3- La recomposition de la classe politique et ses implications dans le champ syndical estudiantin de 1993 à 1999}

Après trente trois années de règne sans partage du PDCI, nous avons assisté à la formation de grands blocs politiques en côte à la faveur du multipartisme. Il y avait d'un côté les partis de l'opposition supplantés par le FPI constitués majoritairement des partis de gauche avec une dominance d’idéologie socialiste. De l'autre côté le PDCI, parti de droite au pouvoir avec une idéologie capitaliste. Avec l'arrivée du RDR sur la scène politique en 1994, les rivalités politiques entre le PDCI et ce parti ont favorisé le rapprochement entre le RDR et le FPI. Ce rapprochement a abouti à la formation d'une union entre ces deux partis pour former le Front Républicain dont l’objectif principal était d'évincer Henry Konan Bédié du pouvoir. La création du front républicain est déterminante dans l'histoire de la violence estudiantine. A partir de ce moment, la situation sociopolitique se précarise davantage avec le caractère «ethniciste » que prend l'opposition entre Alassane Dramane Ouattara et Henry Konan Bédié. Cette ethnicisassion a dégradé les rapports entre le groupe Akan dominé par l'ethnie baoulé et le groupe ethnique Mandé nord dominé par l'ethnie malinké communément appelé « Dioula ».

Face à l'ambition politique du président du RDR qui représentait de plus en plus une menace politique pour Henry Konan Bédié, le régime PDCI développe le concept d'ivoirité pour distinguer le vrai ivoirien du faux ivoirien. Comme le dit Milhaud (2006 :2) se référant à Bouquet, le rejet de l'autre graduellement institué comme principe politique à travers l'ivoirité. Ainsi, il entreprend une vaste campagne pour prouver à la communauté nationale et internationale que le président du RDR est étranger en vue de l'écarter de la compétition électorale pour magistrature suprême. Le concept d'ivoirité, au lieu d'être une conception positive de la nationalité ivoirienne et créer un environnement favorable à l'unité nationale, a produit le résultat contraire sous l'effet des diverses interprétations à des fins politiques dont il a fait l'objet. La campagne pour la discréditation du candidat du RDR à 
l'effet de l'éliminer de la compétition électorale ${ }^{64}$ (Gobille, 2009) a d'une part inscrit désormais le président du RDR sur la liste des leaders politiques charismatiques et populaires, mais dans le même temps elle l'a propulsé au devant de la scène politique comme "le symbole de l'exclusion sociale et politique à laquelle se sont progressivement identifiées les populations du nord, ayant le sentiment d'être devenues des citoyens de seconde zone » (Akindes, Fofana \& Koné, 2010 : 93). D’autre part, elle a contribué à la fragilisation de la situation sociopolitique, et le milieu syndical estudiantin n’en n’a pas été épargné.

\section{4- Une autonomisation syndicale manquée}

L'entrée en scène de la fesci en 1990 s'inscrit dans un contexte d'âpreté de la compétition entre les partis politiques pour le contrôle de l'espace universitaire par le canal des associations syndicales estudiantines. Son activisme dès le départ visait à insuffler un nouveau dynamisme au mouvement revendicatif syndical estudiantin à travers un renouveau syndical caractérisé par son radicalisme que les militants qualifient de lutte révolutionnaire. Ce renouveau syndical avait pour objectif de départ de mettre fin au mode de syndicalisme exercé par le meeci qui selon les militants de la fesci faisaient du "syndicalisme de participation ». Pour la fesci, le meeci était aux ordres du PDCI, et faisait plus de la politique que de la revendication syndicale pour la cause des étudiants. Ce renouveau syndical visait donc à dissocier l'activité politique de la lutte revendicative syndicale estudiantine, par conséquent rendre les syndicats autonomes des partis politiques. Cependant, ce syndicat n'avait pas d'autonomie financière pour son fonctionnement et la réalisation de ses activités. Elle était financièrement dépendante des partis politiques. Ce renouveau syndical distant de la politique n'avait à n'en point douter aucune chance d'échapper à la récupération politique ou à sa mutation en une lutte politique pour une double raison. D'une part, les militants des syndicats sont convaincus que sans moyens financiers leurs actions ne peuvent prospérer et atteindre les objectifs et que les partis politiques sont la principale source de financement de leurs activités. D'autre part, les conditions de de naissance de la fesci porteuse de ce projet de renouveau syndical. Dès lors, il est évident que le

${ }^{64}$ Gobille «Le « et » mettrait hors course la candidature du leader du RDR, Alassane Dramane Ouattara, dont la nationalité ivoirienne est régulièrement mise en cause depuis plusieurs années. Mais il en va, au-delà du cas particulier d'Alassane Ouattara, de la question de l'identité nationale en Côte d'Ivoire. Le chef de la junte alors au pouvoir, le général Robert Guéi, sous la pression et parce qu'il pense y avoir intérêt, opte alors pour la conjonction « et ». Il inscrit ainsi ce qui a été appelé l' « ivoirité » au cœur même de la Constitution. La candidature à l'élection présidentielled'Alassane Ouattara est alors invalidée, et avec elle l'exclusion des populations du Nord, dont il est originaire, de la communauté nationale, est institutionnalisée et intériorisée, p1 
renouveau syndical tant recherché était mort-né. La fesci est née dans un contexte politiquement hardi qui a favorisé et facilité son rapprochement des partis politiques dès le départ. A travers son slogan « il n’a y a pas d'école véritable sans démocratie », la fesci exprime sa volonté de contribuer à la mise en place d'un cadre d'expression plurielle et libre. Dans le contexte d'éclosion du pluralisme politique, les discours allant dans le sens de l'instauration d'un cadre de liberté d'expression, d'action, d'association pour la dénonciation du mode de gestion du pouvoir par le régime PDCI, du soutien ou de la compassion à la condition de précarité socio-économique des étudiants ne pouvaient qu'être soutenus par la conscience collective. Ces discours qui ont eu un écho favorable auprès des étudiants, ont donc contribué à l'impulsion et la popularité de la fesci. Par contre ils ont contribué à l'impopularité et à l'affaiblissement du meeci, allant jusqu'à sa disparation progressive. Le leader syndical sous l'ère du parti unique étant détrôné de son statut de leader à l'aube du multipartisme, la fesci devient le syndicat populaire dans les établissements universités avec l'adhésion massive des étudiants à sa cause. L’unesci, deuxième syndicat né en 1997 pour opposer à la fesci un équilibre de force n’a pas échappé à ce soutien politique dès sa naissance.

Dans sa logique d'exercer son leadership sans partage et sans vouloir le perdre, l'arrivée de l'unesci avec son slogan « oui au dialogue, à la concertation et la négociation, non à la violence » a été qualifiée par la fesci de provocateur et interprétée comme un retour « déguisé » du meeci dans sa volonté affichée de reconquérir son leadership pour le contrôle du monde scolaire et universitaire au profit du PDCI. Dès lors, nous constatons un leadership syndical s'exprimer sur fond d'opposition politique à la faveur de l'ouverture du champ politique qui a favorisé l'entrée massive de la jeunesse dans l'arène politique. Le renouveau syndical recherché par la fesci en rompant avec la pratique syndicale assujettie à la politique n’a donc pu être réellement effectif.

Ainsi, à l’image de la classe politique caractérisée par une divergence d’opinions et d'idéologies pour la gestion de la nation, les syndicats créés à la faveur du multipartisme voulaient au départ contribuer à l'amélioration du système éducatif en Côte d'Ivoire, dans la diversité d’opinion et d'idéologie, et dans la complémentarité. Par conséquent contribuer au renforcement de la démocratie dans l'intérêt des élèves et étudiants. Toutefois, l'exercice de ce pluralisme syndical dans le milieu associatif estudiantin se fait sur fond de violence. Cette violence qui opposait les syndicats entre eux, mais aussi les militants d'un même syndicat, s'est intensifiée au fil des années, et a finalement intégré le fonctionnement des syndicats et les habitudes des militants syndicalistes. 


\section{III- La perception des syndicats de la violence}

La compréhension de la perception des syndicats de leur usage de la violence passe par la connaissance des sources de légalité et de légitimité de la violence d'une part, et le lien entre l'exercice du syndicalisme et la politique d'autre part. Toutefois, il importe de clarifier ce que nous entendons par sources de légalité et de légitimité. Il s'agit de montrer ici ce que les syndicats définissent comme sources légales et légitime qui les autorisent à faire usage de la violence, même si celles-ci ne sont pas conformes aux lois de l'institution universitaire.

\section{1- Les sources de légalité et de légitimité de la violence syndicale estudiantine}

Deux sources de légalité de la violence sont à relever pour les syndicats. Il s'agit de la source statutaire avec l'autorisation de l'usage de la violence dans les statuts et règlements intérieurs de certains syndicats, et de la source non statutaire basée sur du mimétisme pour d'autres syndicats. En effet, convaincus du pouvoir conféré par la violence, plusieurs syndicats en font usage bien qu'aucune disposition de leur statut et règlement intérieur ne les y autorise. Pour les syndicats, la légitimité de la violence s'explique par le fait qu'elle est l'ultime recours après l'échec du dialogue. Que fait-il entendre par l'échec du dialogue? Pour les syndicats, l'inflexibilité des autorités ou de l'interlocuteur est un échec du dialogue. La réussite du dialogue suppose l'atteinte des objectifs liés à leur revendication.

"Nos textes en son article 4 ou 7 je crois nous autorisent à faire usage de tous moyens en cas d'échec du dialogue pour faire aboutir nos revendications. C'est ce qui fait nous avant d'agir, on discute toujours. Quand on constate qu'il n'y a plus de solution on est obligé d'utiliser autres moyens pour t'obliger à accepter ce qu'on te demande. Les gens quand ils nous voient agir automatiquement ils disent c'est encore la fesci, mais ils ne savent pas qu'avant d'agir là ce qu'on a fait jusqu'à ce qu'on soit obligé d'utiliser la violence ». (Extrait de l'entretien réalisé avec K.D.L en 2015)

"Mais tous les moyens là ça veut dire quoi concrètement, ça veut dire que si peux stresser, si je peux les empêcher de sortir, si je peux les intimider tout ça là. Ce sont des choses qui arrivent. Souvent il arrive qu'on enferme le personnel et on garde la clé, souvent même l'administration et nous là même on se menace, souvent même on peut dégonfler les pneus des enseignants, souvent aussi les casses, c'est-à-dire que c'est l'intimidation. Mais tu ne veux pas résoudre mon problème et on me dit que je peux utiliser tous les moyens à bord pour faire aboutir ma revendication, mais c'est ce que j'ai que j'utilise. Si la négociation a échoué, je passe à une vitesse 
supérieure, pas pour moi-même hein, mais dans l'intérêt de mes camarades étudiants, c'est autorisé par nos textes. Donc tu vois que quand on négocie là et quand on privilégie le dialogue ça évite beaucoup de choses ». (Extrait de l'entretien réalisé en 2015 avec O.K.C)

A partir de ces propos, on assiste à une antinomie de la perception de la violence entre celle des auteurs et celle des autorités administratives et académiques des universités. Conformément aux normes qui régissent les universités, adopter un comportement violent pour s'exprimer est une déviance. Mais pour les auteurs, la violence s'avère une nécessité pour l’intérêt collectif.

\section{2- Le lien entre le syndicalisme et la politique}

Pour les militants des syndicats, le syndicalisme est l'antichambre de la politique, et son exercice ne peut être dissocié de la politique. Il existe donc un lien congénital entre le syndicalisme et la politique par le fait que le syndicalisme conduit à la politique. Partant de cette perception, nous sommes amenés à nous demander si l'ambition politique du militant de syndicat d'étudiant est-elle nourrie dès son adhésion syndicale. Pour les leaders, bien que l'ambition politique prime, mais elle est postérieure à l'acquisition d'un réseau de relation. Contrairement au leader, l'intention politique des militants de base est précédée ou même voilée par l'ambition de construire d'abord un réseau de relation qu'il va capitaliser plutard pour son insertion socioprofessionnelle ou son positionnement politique. Pour y parvenir, l'objectif immédiat est de mériter la confiance et l'estime du leader pour bénéficier d'une promotion syndicale.

«Tu sais, dans le syndicalisme, chacun prépare sa sortie syndicale. Donc tu te fais des relations et après tu peux t'appuyer sur ces relations pour avoir du travail (rire). Mais djo en côte d'Ivoire ici, c'est relation qui fait tout». (Extrait de l'entretien réalisé en 2015 avec $\mathbf{F . Y )}$

\section{IV- Les enjeux de la violence syndicale estudiantine}

\section{1- L'acquisition de pouvoir par la domination des espaces publics}

Face à l'école qui n'offre pas suffisamment de garantie d'obtention d'emplois, pour les syndicats le positionnement politique s'avère une alternative à l'insertion professionnelle. Pour bénéficier de cet avantage politique, le syndicat doit faire la preuve de sa capacité de mobilisation et de domination de son champ d'action. Etant soumis aux contraintes tant sociales qu'économiques, ils s'engagent de plus en plus dans la sphère politique par le biais du syndicalisme à travers la violence comme mode principal de régulation des conflictualités internes et externes. Pour Babo 
(2011), le caractère patriotique du militantisme des jeunes s'offre dès lors comme une opportunité de résorption de leur insécurité sociale qu'ils n'auraient pu régler autrement. Aussi, l'absorption du temps d'étude par l'activité syndicale favorise une sédentarisation au militantisme syndical et contraint les militants à s'orienter vers la politique où le critère d'âge est moins important. A la fin de leurs études, n’ayant pas les diplômes requis pour certains d'entre eux pour exercer en entreprise, la professionnalisation politique s'avère donc pour eux une alternative d'insertion socioprofessionnelle.

j Avec la dynamique des syndicats d'étudiant, l'espace universitaire devient un espace de concentration d'énergie des partis politiques dans le but de les contrôler par l'intermédiaire des syndicats. Les avantages politiques liés au contrôle de l'espace universitaire est source de violence pour le statut de leader. Ce qui favorise la cristallisation des violences syndicales estudiantines autour du leadership. Les syndicats sont conscients que le statut de leader confère des avantages et des traitements de faveur, que le contrôle des espaces publics est devenu une ressource conférant le pouvoir. Le contrôle de ces espaces publics notamment les établissements scolaires et universitaires faisait donc des objectifs des syndicats. L'occupation et la domination de l'espace universitaire par les syndicats notamment la fesci se sont faites progressivement. Cette occupation a commencé avec l'occupation des amphithéâtres à travers la stratégie de son positionnement par le biais de ses délégués dans les amphis en tant que représentants des étudiants dans les UFR. Les amphis et résidences universitaires sont devenus la chasse-gardée des syndicats supplantés par la fesci. Sous l'effet de l'impunité, l'autorité des syndicats s'est étendue sur tout l'espace universitaire. Pour les autres syndicats, la conquête de cet espace en vue de le contrôler était un objectif à atteindre. A la faveur de la crise de 2002 qui a fragilité l'autorité de l'Etat, cette fragilité dans les universités a été transformée par les syndicats en opportunité par la déstructuration de l'espace universitaire en le transformant en un lieu de rente du désordre sous le couvert du syndicalisme patriotique qui rend légale la violence qui en résulte. Dès lors, le militantisme syndical s'offre dès lors comme une opportunité de résorption de leur insécurité sociale qu'ils n’auraient pu régler autrement. Pour la jeunesse estudiantine à l'image de la jeunesse ivoirienne en général, l'usage de la violence se présente à la fois comme une stratégie d'affirmation de leur situation de crise, et un moyen de domination et de positionnement.

\section{2- La stratégie de domination du champ universitaire}

Durant la décennie 2000-2010, le contrôle du champ universitaire échappait aux autorités administratives et académiques au profit des syndicats d'étudiants, notamment la fesci. Par ce contrôle, la fesci démontre 
qu'elle a la maîtrise de la mobilisation qu'elle utilise contre ou pour tout régime politique. La maîtrise de cette compétence était un objectif à atteindre pour les autres syndicats. En référence à Fourboul dans la typologie des zones d'incertitude, cette compétence acquise est une zone d'incertitude que la fesci maîtrise. En effet, il y a d'autres types de situation qui ne renvoient à aucune source de légitimité et qui sont par elle-même créatrices de pouvoir. Ce sont celles dans lesquelles l'individu parvient à maîtriser ce que Fourboul appelle une zone d'incertitude. Ces zones d'incertitude constituent des atouts pour l'individu. Il va essayer d'en accroître le nombre et l'étendue (Fourboul, 2013). Par la maîtrise de cette compétence, la fesci a su se positionner comme un acteur incontournable dans le jeu politique. Le contrôle a commencé par l'occupation des amphis et des résidences estudiantines. L’occupation des amphis à commercé avec la crise des machettes. Lorsque Blé Goudé a su qu'il n’était pas dominant sur les campus lors des campagnes pour la succession de Soro Guillaume, après son élection à la tête de la fesci, il a fait le choix du contrôle des amphis un de ses objectifs à atteindre. Cela a été facilité avec l'arrivée du FPI au pouvoir et la crise de 2002. L’objectif visé par le contrôle des amphis était de rompre avec un ordre social qu'il qualifiait de «fortement hiérarchisé » et qui les reléguait au second plan ou qui les ignorait. Dans le but de restaurer une gouvernance universitaire en comptant parmi les acteurs de prise de décisions dans les universités, ce contrôle s’avérait une nécessité. La volonté de dominer l'espace universitaire est donc la conséquence de la perception que les syndicats projettent sur euxmêmes dans le champ universitaire. En effet, ils jettent sur eux-mêmes l'image d'acteurs passifs dans les prises de décisions dans les universités. C'est donc pour les syndicats l'expression de leur volonté de changement d'une situation qui perdure et instaurer un cadre participatif dans les prises de décisions dans les universités. Mais, un cadre participatif dans lequel ils souhaitent être activement impliqués. Nous sommes donc dans une démarche syndicale qui vise à rompre avec la relation de domination intergénérationnelle telle qu'analysée par Akindes dans un cadre global. En effet selon Akindes (2011 : 3), "la jeunesse ivoirienne déploie deux types de stratégies pour pouvoir évoluer ou inverser le sens de la domination qui est intergénérationnelle, en procédant d'abord par la négociation qui n'importe guère le détour de la violence, dans l'espoir que cette démarche aboutisse, avant de passer à une phase violente qu'ils qualifient de réinvention de soi dans la violence en empruntant le détour de le brutalité ». La démarche syndicale s'inscrit dans une telle stratégie selon nos enquêtés. Elle consiste pour les syndicats à épuiser toutes les voies du dialogue dans l'espoir qu'elles aboutissent. Le recours à la violence est donc consécutif à l'échec du dialogue. L'usage de cette violence est donc pour eux un moyen de coercition pour l'atteinte des résultats attendus de la revendication. Un 
moyen qui leur permet donc d’imposer aux autorités leurs desiderata en les contraignant à satisfaire à leurs besoins.

«Tu sais, l'administration trouvait qu'on était gênant, donc elle voulait nous contourner et travailler directement avec les délégués d'amphi. Quand c'est comme ça, on ne va plus parler de syndicat. Nous on a compris ça. Donc nous aussi on a décidés de positionner nos gars labà et on fait tout pour qu'ils soient élus. Au cours des réunions, des délégués, ils sont surpris de voir nos gars. Mais ils ne peuvent rien dire, puisqu'ils ne sont pas là en tant que fesci, mais en tant que délégués d'amphis. Quand c'est comme ça aucune information ne nous échappe ». (Extrait de l'entretien réalisé avec OCK en 2015)

\section{3- La violence: un indicateur du dynamisme syndical pourun positionnement politique}

Le changement de régime avec l'arrivée du FPI au pouvoir marque un évènement de l'évolution de l'histoire syndicale estudiantine. Après avoir affiché son soutien au Président Gbagbo au cours de l’élection présidentielle d'octobre 2000, la fesci ne craint plus d'être victime des violences d'Etat avec l'arrivée du FPI au pouvoir. La lutte syndicalise s'est radicalisée davantage avec la guerre de 2002, avec la volonté affichée de la fesci de soutenir le pouvoir de Laurent Gbagbo et sauver la démocratie, en s’opposant par tous les moyens aux membres de la rébellion et à tous ceux qu'elle soupçonnait d'être proches ou affiliés à la rébellion. Elle radicalise donc sa position sur le campus et devient plus violente à l'égard des autres syndicats. Restée fidèle dans sa logique de soutenir et défendre le régime de Laurent Gbagbo, la fesci suit majoritairement les mots d'ordre de soutien au régime FPI. Jouant les avant-gardistes de la lutte contre la rébellion, la fesci s'érige en agent de sécurité de l'espace universitaire sous son contrôle et en a profité pour se positionner au cœur du système en tant qu'acteur incontournable. Dès lors, elle devient un acteur stratégique politique pour le FPI. Elle en profite pour exercer son influence et sa domination sur les campus et résidences universitaires pour en faire des opportunités à la fois politiques et économiques, et s'est inscrite dans une logique de gestion patrimoniale de ces espaces. Comme le dit Kouassi (2013), la fesci a été un acteur central des différentes crises qu'a connues la Côte d’Ivoire depuis son avènement sur la scène politique en 1990. Tous les secrétaires généraux de la fesci ont été des acteurs de premier plan de la scène politique en Côte d’Ivoire. La fesci a été un champ d’expérimentation des stratégies politiques des partis, de la violence politique et finalement de la violence armée avec Soro Guillaume, chef de la rébellion qui a divisé le pays en deux. Si cette affirmation de Kouassi relève d'un constat si évident, toutefois, les crises 
politiques de 1990 ne sont survenues ex nihilo. Elles ont pour origine la crise économique, facteur d'exacerbation de la crise politique. Dans le contexte délétère d'amorce du multipartisme empreint de violences politiques généralisées, les étudiants ivoiriens à l'instar de ceux des autres pays africains ont été aux avant-postes de la contestation démocratique. L'aspiration à la liberté d'expression et au mieux être économique et social mis en avant, génère sur les campus universitaires l'émergence des associations syndicales qui servent de porte-paroles des partis politiques (Dechi, 2006). Cette posture d'avangardiste des étudiants suscitée et soutenue par les partis politiques de l'opposition et certaines organisations de défense et de protection des droits de l'homme, visait à mettre fin au monolithisme qui s'exerçait dans le milieu associatif estudiantin et rendre effectif le pluralisme syndical à l'image du multipartisme politique. Cette dynamique associative estudiantine marquant un éveil de la jeunesse en vue de l'instauration de la démocratie n’était pas un phénomène propre à la Côte d'Ivoire. C'était une tradition de lutte pour les pays africains surtout les pays africains francophones bien avant leur accession à l'indépendance (N'goran, 2012). Cette tradition consistait à occuper l'arène politique en arguant du prétexte syndical en milieu scolaire. L'exercice syndical dans ce contexte d'amorce de la démocratie concurrentielle tout en suivant la même logique que celle du système monopartite, prend une nouvelle dimension en se radicalisant avec l'introduction des armes dans la lutte syndicale.

\section{Conclusion}

L’espace syndical estudiantin a enrégistré la naissance de plusieurs syndicats, ce qui à priori est un indicateur de l'expression démocratique. Toutefois, ce «libéralisme » syndical estudiantin s’est manifesté par la violence exercée à la fois sur les étudiants et les personnel administratif et académique durant les deux décennies qui après l'indépendance. Avec le départ nouveau qui est l'expression d'une rupture avec les pratiques qui ont favorisé la perte du lustre d'antan des universités ivoiriennes jadis réputées pour être des cadres de formation de qualité, on assiste à survivance de cette violence. Il s’agit donc d'expliquer la survivance de ce phénomène à partir de l'analyse de la perception que les syndicats ont de leur usage systématique de la violence comme moyen d'expression ainsi que des enjeux qui sont liés à la violence. L'étude nous permet de dire que les syndicats ont toujours été au cœur de la politique en Côte d'Ivoire et constituent des acteurs stratégiques pour les partis politiques pour le contrôle de la jeunesse estudiantine et scolaire dans leur stratégie de conquête du pouvoir exécutif. Les syndicats ont donc pris conscience de leur position d'acteurs stratégiques pour les partis politiques en qu'ils ont su transformer pour certains cette position d'acteur stratégique en opportunité de positionnement politique et 
d'insertion professionnelle. Pour d'autres la transformation de cette position en opportunité était un objectif à atteindre. Dans un contexte de désillusion sociale très vite capitalisée par les partis politiques, la voie de la politique était pour les syndicats une alternative à l'emploi. Dans cette perspective, la domination des espaces publics tels que les universités conférait du pouvoir et était un atout pour atteindre cet objectif.

\section{References:}

1. AKINDES, F. (2000). «Inégalités sociales et régulation politique en côte. D'ivoire. La paupérisation en Côte d'Ivoire est-elle réversible ? »Politique africaine $N^{\circ}$ 78. Karthala.pp126-141.

2. AKINDES, F., FOFANA, M., \& KONE, G. (2010). « Côte d'Ivoire : insurrection et contre-insurrection ». Alternatives sud, Vol.17. pp 93-99. http://www.cetri.be.

3. AKINDES, F. (2011). "La Côte d'Ivoire depuis 1993: La réinvention risquée d'une nation ». pp1-38. http://www.codesria.org.

4. BABO, A. (2011). «Les enjeux de la violence politique chez les milices des jeunes patriotes d'Abidjan en Côte d'Ivoire ».ann.univ.de lomé, sér.lett, tome xxxi-1. pp247-260.

5. DECHI, A.G. (2006). «La lutte contre la violence en milieu universitaire ivoirien $» . \quad$ Edit CERAP, http://www.memoireonline.com/a/fr/cart/show.

6. DRAUS, F. (1984). "Raymond Aron et la politique». Revue française de science politique, 34 année, $\mathrm{N}^{\circ} 6 . p p 1198-1210$.

7. FOURBOUL, C.V. (2013). "Pouvoir : jeux de pouvoir et analyse stratégique ». pp1-11.http://voynnetf.fr.

8. GOBILLE, B. (2009). « Comment la stabilité politique se défait-elle ? La fabrique de la dépacification en Côte d'Ivoire, 1990-2000 ». Congrès AFSP, Section thématique, $\mathrm{N}^{\circ} 44 . p p 1-57$.

9. GOIN BI, Z.T. (2010). « Déchainer les libertés académiques en Côte d'Ivoire une responsabilité des universitaires ». pp111.http://www.codesria.org/.

10. HUMAN, R.W. (2008). " Le militantisme étudiant dans les années 1990 ; de la clandestinité au schisme politique ", http://hrw.org/french/backrouder.

11. HUMAN, R.W. (2008). «La meilleure école: la violence estudiantine, l'impunité et la crise en Côte d'Ivoire», http://hrw.org/french.

12. HYDEN, G. (1992). "Gouvernance et étude de la politique: Gouverner l'Afrique, vers un partage des rôles ». Colorado, Nouveaux Horizons. 
13. KONATE, Y. (2003). «Les enfants de la balle. De la Fesci aux mouvements de patriote ». Politique africaine, N89. pp49-70.

14. KONE, G. (2011). « Logiques sociales et politiques des pillages et barrages dans la crise postélectorale en Côte d'Ivoire ». Politique africaine, $\mathrm{N}^{\circ} 122 . p p 145-160$.

15. KOUADIO, M.K.D. (2010). « La question controversée des libertés syndicales en milieu universitaire en Afrique: le cas du syndicalisme estudiantin en Côte d'Ivoire, constat d’un déni de liberté académique notamment les libertés dans les universités publiques en côte d'Ivoire ».pp1-21. http://www.codesria.org.

16. KOUASSI, K. S. (2013). "Regard retro-prospectif sur les crises ivoiriennes de 1993 a 2011 ». Cinq Continents Vol 3, N8, pp127149.

17. MEL, M.R. (2009). « Anthropologie des enjeux de la violence chez les lagunaires de Côte d'Ivoire ». African sociological review, Cape Town, South Africa. pp78-104.

18. MILHAUD, O. (2006). « Christian Bouquet, Géopolitique de la Côte d'Ivoire. Le désespoir de Kourouma ». CollectionPerspectives géopolitiques, Paris, Armand Colin, 2005. http://www.cafe-geo.net.

19. N'GORAN, K.L. (2011). «violence scolaire : une stratégie de résolution des violences » pp64-75.http://www.cdoc-alpha.be.

20. N'GORAN, D.K. (2012).« les enfants de la lutte : chronique d'une imagination politique à Abidjan ». Editions publibook.

21. N'GORAN, K.P., \& SILUE, N.O. (2012). « modes de participation des jeunes au jeu politique en côte d'ivoire : l'exemple des espaces de discussions de rue ». European Scientific Jounal, Vol 8, N8. pp148167.

22. PROTEAU, L. (1998). « la "reproduction en question" école, université et mouvements sociaux en Côte-D’ivoire ». Curapp questions sensibles, PUF.

23. SEARCH FOR COMMON GROUND. (2013). «Étude de base du Projet Promouvoir une culture de la paix en milieu scolaire en Côte d'Ivoire ». Rapport final, http://www.sfcg.org.

24. VANGA, A.F., KOUAKOU, A., \& SIKA, C. (2006). « La violence à l'école en Côte d'Ivoire, quelle implication des syndicats d'élèves et d'étudiants ». http://www.rocare.org/vangga.pc. 